\title{
Thin film composite polyelectrolyte multilayer nanofiltration membrane fabricated using spin assisted layer by layer assembly: Application of solution diffusion film model
}

\author{
A.M. Alghamdi, F. Fadhillah* \\ Chemical Engineering Department, Al Imam Mohammad Ibn Saud Islamic University, Riyadh, Saudi Arabia, 11432, Saudi Arabia
}

Article history:

Received: 13 May 2020 / Received in revised form: 28 May 2020 / Accepted: 30 May 2020

\begin{abstract}
Thin Film Composite (TFC) Polyelectrolyte Multilayer (PEM) Nanofiltration (NF) membrane consisting of multilayer of Poly(diallyl dimethyl ammonium chloride) (PDAC) and Poly(sodium 4-styrenesulfonate) (PSS) deposited on polyethersulfone (PES) support was prepared using spin-assisted layer by layer assembly (SA-LbL). This work is an effort to discover a new material with better characteristics and performance compared to commercial polyamide (PA) NF membrane. Two main operating conditions, namely temperature and salt concentration as well as their effect on membrane performance were investigated and studied. Solution-diffusion Film Model (SDFM) membrane employing two fitting parameters i.e. membrane salt permeability $\left(\mathrm{P}_{S}\right)$ and stagnant layer salt permeability $\mathrm{P}_{\mathrm{S}}{ }^{(\delta)}$, was used to explain membrane transport across the membrane. The result showed a good agreement between experimental and observed rejection rate suggests PEM membrane to behave similar to typical solution diffusion-type composite membrane and SDFM can be used to predict the membrane performance and behavior.
\end{abstract}

Keywords: Layer-by-layer assembly, polyelectrolyte, membrane transport, solution diffusion model, nanofiltration

\section{Introduction}

Nanofiltration (NF) membrane is a relatively new class of pressure-driven membrane lying between reverse osmosis (RO) and ultrafiltration (UF). It typically has the pore size of 1 $\mathrm{nm}$ corresponding to molecular weight cut-off of 300-500 Da [1]. Owing to its ability to reject any multivalent ions and organic molecules coupled with its relatively higher water permeability compared to RO membrane, NF has progressively gained attention from researchers in the area of membrane science and technology since its appearance in the mid of 1980s (as shown in Figure. 1). The commercial application of NF can be normally found in the removal of multivalent ions [2], heavy metals, dyes [3], pesticides, micropolutants [4] , and textiles [5].

Commercial Polyamide (PA) NF membrane fabricated through interfacial polymerization on the top of polysulfone (PSF) support shows a superior performance in terms of water flux and salt rejection. Having said that, this membrane exhibits low resistance to fouling, particularly biofouling. Researchers have continuously investigated the appropriateness of other materials for NF/RO membrane application. The challenge for fabricating ultra-thin layer of any material is the fabrication method itself. Among thin film fabrication methods, layer by layer (LbL) assembly i.e. dip-LbL [6] has been considered as

* Corresponding author. Tel.: +966-1-125-86293.

Email: fffadhillah@imamu.edu.sa / faridfad.imamu@gmail.com an inexpensive, versatile, robust, and bottom-up nanofabrication technique.

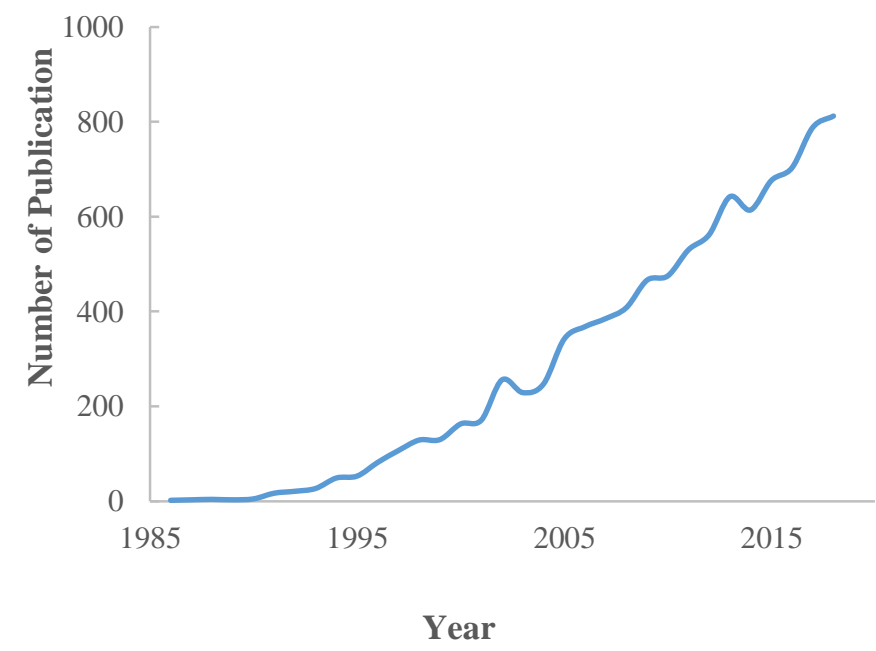

Figure. 1. Number of publications per year related to NF in various applications

The application of dip-LbL assembly for fabricating NF membrane, particularly for ion removal, has been extensively investigated [2,7,16-18,8-15]. Nevertheless, the process of this method is found time consuming, normally requiring several minutes to deposit single layer. In view of this main drawback, 
the method has then been modified into spray-LbL assembly [19] and spin-LbL assembly [20], principally to shorten the deposition time. The suitability of spray and spin-assisted LbL for RO/NF is just recently studied by Jason et al. [21] and Fadhillah et al. [22], respectively.

Our previous work [22] on NF employing two polyelectrolytes, namely poly(diallyl dimethyl ammonium chloride) (PDAC) and Poly(sodium 4-styrenesulfonate) (PSS) deposited on polyethersulfone (PES) support layer showed a promising result. As recently implemented method, membrane transport through this kind of film is still quite unclear. Some researchers suggested that the rejection mechanism of this membrane follows bipolar membrane [23]. However, our own study showed no any significant increase in rejection rate by doubling the number of layers [24]. It was also reported that changing outermost layer results in the significant change of anion flux. Therefore, it is suggested that only uncompensated charges near the film-solution interface bring a significant effect on the ions rejection mechanism [8]. Based on those experimental results, one can assume that the entire membrane actually behave similar to typical NF or RO membrane.

NF membrane transport is considered as a complex process involving many parameters such as size, steric, electric and dielectric hindrance effects as well as electroviscosity [25]. Up to now, two main models have been developed to describe the transport mechanism through the membrane namely irreversible thermodynamics model (ITM) [26] and solution diffusion (SDM) [27]. Among ITM, Donnan-Steric Partitioning Pore Model (DSPM) is considered the most interesting and innovative one [28]. This model is based on Nerst-Plack equation and includes steric/electric exclusion mechanism. Having said that, DSPM is still not able to fit experimental rejection rate of various electrolytes with a single value of the membrane-thickness-to-porosity ratio and failed to describe the high rejection rates observed with some NF membranes in the case of ionic solutions containing divalent counterions such as $\mathrm{Ca}^{2+}$ or $\mathrm{Mg}^{2+}$ [29]. Not to mention, DSPM model requires some membrane characteristics such as pore radius and membrane charge, which is normally not readily available, and the complexity of the model itself, hinder its application in any practical situation.

In contrast, SDM offers the simplicity yet quite robust to be applied for ionic or non ionic species transport through RO and NF membranes. In the previous work [25,30], SDM model was modified by including the coupling effect between ions within the stagnant layer, a thin film adjacent to membrane surface in which concentration polarization occurs. This model, so-called, solution diffusion film model (SDFM) considers the solutes (uncharged molecules or individual ions) transport occurs via diffusion, electric migration and, to some extent, convection through both stagnant layer and membrane. Those solutes travel across both layers at different speed but the electroneutrality must always be maintained everywhere.

The objective of this paper is to study the effect of operating condition on the performance of PDAC/PSS NF membrane prepared using SA-LbL assembly. Two main observable parameters namely observed rejection rate $\left(R_{\mathrm{obs}}\right)$ and permeate flux $\left(\mathbf{J}_{\mathrm{v}}\right)$ will be measured and analyzed using SDFM. This work also aims to validate SDFM in PEM membrane using single salt feed in a prescribed condition.

\section{Materials and Methods}

\subsection{Materials}

In this work, PDAC (molecular weights of 400,000$500,000)$ in the form of $20 \mathrm{wt} \%$ in $\mathrm{H}_{2} \mathrm{O}$ solutions and PSS (molecular weights of 70,000) in the form of $30 \mathrm{wt} \%$ in $\mathrm{H}_{2} \mathrm{O}$ solution were purchased from Sigma-Aldrich (USA). Both polyelectrolytes were used as received without purification. Extra-pure $\mathrm{NaCl}$ was obtained from Scharlau (Spain) while extra-pure $\mathrm{MgCl}_{2} \cdot 6 \mathrm{H}_{2} \mathrm{O}$ was obtained from Loba Chemie Pvt. Ltd (India).

The stock solutions of the polyelectrolytes (PDAC and PSS) with polyelectrolyte concentration of $0.02 \mathrm{M}$ were prepared. $\mathrm{NaCl}$ was also added to both solution at a concentration of 0.05 $\mathrm{M}$. The $\mathrm{pH}$ of the PDAC solution was adjusted to 4.9 while the $\mathrm{pH}$ of the PSS solution was kept at 8.2. PES ultrafiltration (UF) membranes (YMPWSP3001, Sterlitech Corp., USA) with a pore size corresponding to a molecular weight cut-off of 10 $\mathrm{kDa}$ were used as the support layers for fabricating the PEM TFC membranes. The PES UF membranes were tested and were found to exhibit a permeation flux of $235.7 \mathrm{~L} / \mathrm{m}^{2} \cdot \mathrm{h}$ at a pressure of 10 bar and temperature of $33.5^{\circ} \mathrm{C}$. Figure 2 shows the molecular structures of the various materials used.

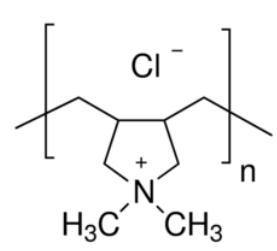

(a)<smiles>CC(C)(C)CC(c1ccc(S(=O)(=O)O)cc1)C(C)(C)C</smiles>

(b)

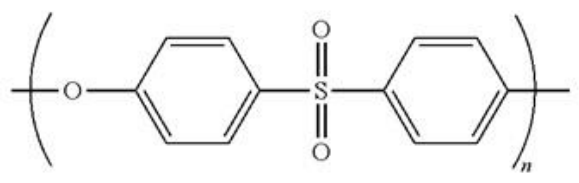

(c)

Fig 2. Molecular structures of materials used to synthesize PEM TFC membranes: (a) PDAC, (b) PSS, and (c) PES

\subsection{Fabrication of PEM TFC Membrane}

The PDAC and PSS solutions were alternately deposited on the PES UF membranes using a fully automatic spin coater (POLOS SPIN150i®, SPS Inc.). Prior to deposition, the PES UF support membrane was pretreated in an air plasma using a plasma cleaner (PDC-32G-2, Harrick Plasma Inc) as per a procedure as described elsewhere [31]. After the pretreatment, the PES support became clean, highly negatively charged, and more hydrophilic and was thus ready to be used as a support for LbL assembly.

PDAC was firstly deposited on the spinning PES support. The deposition process was performed at a speed of $3000 \mathrm{rpm}$ [32] with a deposition rate of $0.2 \mathrm{~mL} / \mathrm{s}$ for $10 \mathrm{~s}$. The film was then spin-dried for 20s and then followed with rinsing step using distilled water. PSS was subsequently deposited in a similar manner to PDAC including the rinsing step. A complete cycle comprised of single PDAC and PSS deposition. This 
cycle was then repeated up to 35 times based on our previous work.

\subsection{Permeation test}

Permeation tests were performed using a cross-flow permeation cell (Innovator ${ }^{\circledR} \mathrm{CF} 016$, Sterlitech Corporation) as shown schematically in Fig 3. CF016 system had an active membrane surface area of $20.6 \mathrm{~cm}^{2}$. In this experiment, operating condition such as, temperature and $\mathrm{MgCl}_{2}$ concentration of the feed solution became the object of study and varied. The pressure, temperature, $\mathrm{pH}$, and $\mathrm{MgCl}_{2}$ concentration of feed solution were all kept constant at 10 bar, $32^{\circ} \mathrm{C}, 6.4$, and $500 \mathrm{ppm}$, respectively during the course of permeation test except when the effect of certain parameter was investigated. For instance, when the effect of temperature of feed solution was investigated, it was varied at 20,30 and $40^{\circ} \mathrm{C}$. While, when the effect of $\mathrm{MgCl}_{2}$ concentration of the feed solution was examined, it was varied at 500, 1000, and 4000 ppm.

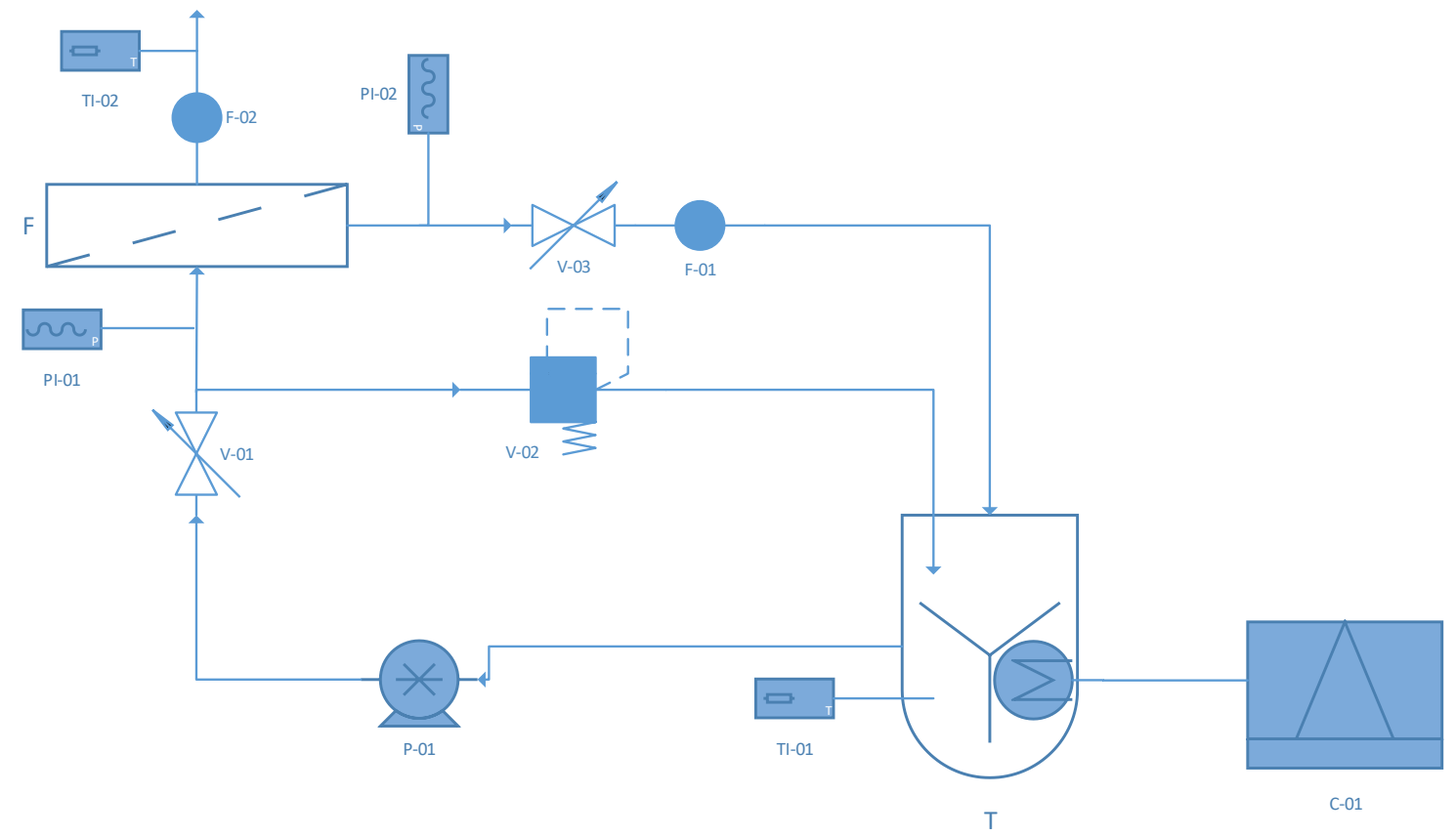

Fig 3. Schematic of permeation system used (F: permeation cell; T: feed tank; C-01: chiller; V-01/03: needle valves; V-02: pressure-release valve; P-01: pump; F-01/02: flowmeters; PI-01/02: pressure gauges; F-01/02: flowmeters; TI-01/02: thermometers)

These tests were performed for 5 hours, and the membrane parameters were measured every hour. Prior to the permeation tests, the membranes were immersed in water overnight for equilibration under wet conditions and to minimize any defects in the membranes resulted from dry conditions.

Several important membrane performance indicators i.e. permeate flux $\left(\mathbf{J}_{\mathrm{v}}\right)$, solute concentration at feed $\left(C_{S}^{(F)}\right)$ and permeate side $\left(C_{S}^{(P)}\right)$ were all recorded. Observable rejection rate $\left(R_{S}^{o b s}\right)$ was then calculated using the following formula:

$$
R_{S}^{o b s}=1-\frac{c_{s}^{(P)}}{c_{s}^{(F)}}
$$

where, $\mathrm{c}_{\mathrm{s}}^{(\mathrm{P})}$ and $\mathrm{c}_{\mathrm{s}}^{(\mathrm{F})}$ are $\mathrm{MgCl}_{2}$ concentration (ppm) for permeate and feed, respectively.

\subsection{Model Development}

The derivation of SDFM was done by Yaroshchuk et al. [30] and Perez et al. [25] for modeling the transport of single dominant salt and trace ion through NF membrane taking transport in a stagnant layer into account. Solute transport within a given thickness of stagnant layer occurred by diffusion, electrostatic migration and convection. The concentration of solute in bulk of stirred liquid was assumed to be homogenous due to complete mixing. It is also important to note that although there was a gradual concentration transition from being stirred to stagnant liquid. In this model, it was assumed to be a sharp transition. The ion transport equations can be written as follows:

$$
J_{i}=-\mathfrak{D}_{i}^{(\delta)}\left(\frac{d c_{i}}{d x}+Z_{i} c_{i} \frac{d \varphi}{d x}\right)+c_{i} j_{v}
$$

where $J_{i}$ is the trans-membrane solute fluxes $\left(\mathrm{mol} / \mathrm{m}^{2} \cdot \mathrm{s}\right)$; $\mathfrak{D}_{i}^{(\boldsymbol{\delta})}$ is the effective diffusion coefficient of solutes in stagnant layer. $\varphi$ is virtual electrostatic potential, $Z_{i}$ is its charge, $c_{i}$ is the virtual concentration of this solute, and $x$ is the trans-membrane coordinate.

From the above model, one can obtain intrinsic rejection rate $\left(\boldsymbol{R}_{\boldsymbol{s}}^{\text {int }}\right)$ as follows

$$
R_{s}^{i n t}=\frac{\left(J_{v} \cdot L / \mathscr{D}_{s}\right)}{1+\left(J_{v} \cdot L / \mathfrak{D}_{s}\right)}
$$

Meanwhile, observed rejection rate was related to intrinsic rejection rate with transport in stagnant layer taken into account as follows:

$$
\left.\frac{1-R_{S}^{o b s}}{R_{S}^{o b s}}=\frac{1-R_{S}^{i n t}}{R_{S}^{\text {int }}} e^{\left(J_{v} \cdot \delta\right.} / \mathcal{D}_{S}^{(\delta)}\right)
$$


Solute diffusion permeability can be expressed:

$$
\begin{aligned}
& P_{S}=\frac{\mathfrak{D}_{S}}{L} \\
& P_{S}^{\delta}=\frac{\mathfrak{D}_{S}^{(\delta)}}{\delta}
\end{aligned}
$$

By combining all equations from (3) to (6), one can obtain

$$
R_{S}^{o b s}=\frac{\left(J v / P_{S}\right) \cdot e^{\left(-J v / P_{S}^{(\delta)}\right)}}{1+\left(J_{v} / P_{S}\right) \cdot e^{\left(-J v / P_{S}^{(\delta)}\right)}}
$$

Observed rejection rate in equation (1) will be fitted with equation (7).

\section{Results and Discussion}

TFC membranes consisting of multilayers of PDAC/PSS on PES UF support were successfully fabricated using SA-LbL assembly. Both PDAC and PSS were considered strong polyelectrolytes whose degree of ionization remained constant over a wide range of $\mathrm{pH}$ values [33-35]. Similar to typical TFC membrane, multilayers of PDAC/PSS acted as an active layer whereas PES UF support afforded the mechanical strength to the entire membrane structure. The evidences of this layer have been discussed in our previous work published elsewhere [22]. As mentioned earlier, in this work, we presented the effect of two main operational parameters (temperature and $\mathrm{MgCl}_{2}$ concentration of feed solution) on the performance of PEM TFC NF membrane.

SDFM was used to investigate the membrane transport across the membrane. Two fitting parameters, i.e. $\mathrm{P}_{\mathrm{S}}$ and $\mathrm{P}_{\mathrm{S}}{ }^{(\delta)}$ used in SDFM were obtained using solver.xlm. General Reduced Gradient (GRC) nonlinear algorithm with multistart method was selected to minimize the differences between experimental and calculated observed rejection rate

\subsection{Effect of Temperature}

Table 1 shows the result of simulation using solver.xlm on two permeabilities as fitting parameters for Eq. 7. The table Table 1 implies that the resistance to the salt transport caused by membrane layer is more pronounce than that of the stagnant layer at the adjacency of membrane surface. In addition, it can be seen that stagnant layer salt permeability $\left(\mathbf{P}_{\mathbf{s}}{ }^{(\delta)}\right)$ was practically constant during the experiment although the temperature was increased from 20 to $40^{\circ} \mathrm{C}$; whereas membrane salt permeability $\left(\mathbf{P}_{\mathbf{S}}\right)$ increased.

Table 1. The effect of Temperature on Membrane Salt Permeabilities, $\left(\mathbf{P}_{\mathbf{S}}\right)$ and Stagnant Layer Salt Permeabilities, $\left(\mathbf{P}_{\mathbf{S}}{ }^{(\boldsymbol{\delta})}\right)$ obtained as fitting parameters for Eq. 7

\begin{tabular}{lcc}
\hline Temperature, ${ }^{\circ} \mathrm{C}$ & $\begin{array}{c}\mathrm{P}_{\mathrm{S}}, \\
\mu \mathrm{m} / \mathrm{s}\end{array}$ & $\begin{array}{c}\mathrm{P}_{\mathrm{S}}{ }^{(\delta)} \\
\mu \mathrm{m} / \mathrm{s}\end{array}$ \\
\hline $20.77 \pm 0.15$ & 0.345 & 57.019 \\
$30.40 \pm 1.13$ & 0.437 & 57.634 \\
$39.15 \pm 0.78$ & 0.629 & 56.121 \\
\hline
\end{tabular}

Constant stagnant layer salt permeability $\left(\mathbf{P}_{\mathbf{s}}{ }^{(\boldsymbol{\delta})}\right)$ can be explained as follows. It is generally known that the stagnant layer, so called concentration polarization layer, is affected by many factors including driving force, membrane characteristics, osmotic pressure and hydrodynamics (cross flow velocity and turbulence) [36]. When the temperature was increased, the PEM underwent a structural change and caused swelling as reported somewhere else $[37,38]$. The membrane swelling at higher temperature was the result of the increase of penetrant i.e. salt and water solubility in the membrane material. The structural change produced a rougher surface that in turn increased the thickness of stagnant layer because more pronounce drag force to the shear flow was imposed. Therefore, although the diffusivity coefficient was proportional to the temperature, its increase was compensated by the increase of stagnant layer thickness that in the end resulted in the relatively constant permeability. Meanwhile, the increase of penetrant solubility in membrane material also increased its concentration especially in the membrane side adjacent to membrane-feed interface that brings the enhancement of penetrant diffusion inside the membrane. This phenomenon is also clearly depicted in Table 1 by the increase of the permeability $\left(\mathbf{P}_{\mathbf{S}}\right)$.

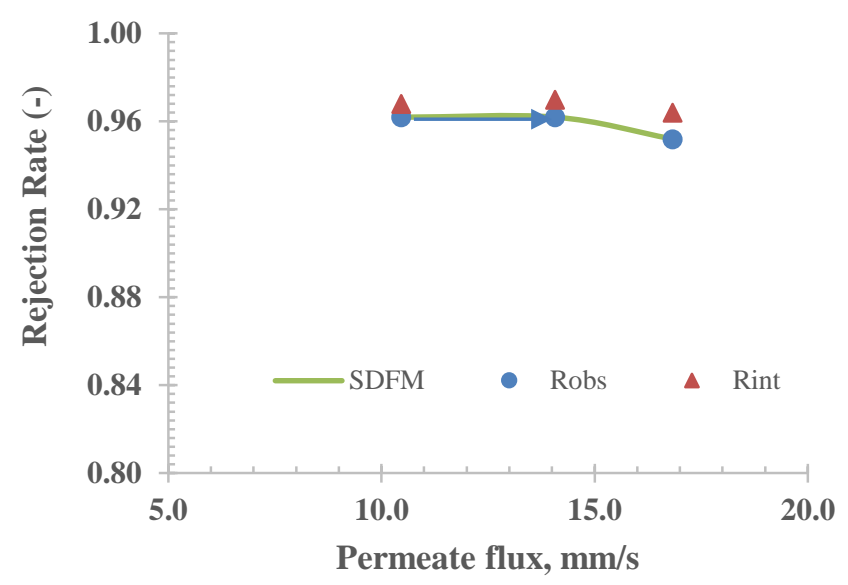

Fig 4. Fitting of Observable Rejection Rate experimental (circle) and intrinsic rejection rate (triangular) with SDFM (continuous line). (Testing condition: $\mathrm{P}$ $=10 \mathrm{bar}, \mathrm{CFV}=0.65 \mathrm{~m} / \mathrm{s}, \mathrm{MgCl}_{2}$ concentration $=607.07 \pm 35.23 \mathrm{ppm}$, and $\left.\mathrm{pH}=6.42 \pm 0.17, \mathrm{~T}=20-40^{\circ} \mathrm{C}\right)$

Fig 4 shows a noticeable difference between the observable rejection and intrinsic rejection rate implying the existence of stagnant layer on the membrane surface. The implication of the difference between the two suggests the increase of salt concentration at vicinity of the surface. In this ultra-thin layer, there was an increase of salt concentration by $19 \%, 27 \%$ and $33 \%$ respectively from corresponding salt concentration at bulk feed, $\mathrm{C}_{\mathrm{S}}{ }^{(\mathrm{F})}$.

The figure also depicts the effect of temperature on membrane performance. As the temperature increased (as shown from the direction of the arrow), the permeate flux also increased owing to the increase of water diffusion across the membrane. Meanwhile, the rejection rate slightly decreased due to the increase of salt diffusion as depicted in Table 1 (shown by the increase of membrane salt permeabilities, Ps).

\subsection{Effect of $\mathrm{MgCl}_{2}$ Concentration in feed solution}

Similar to previous analysis, Table 2 shows two permeability as fitting parameters for Eq. 7 with respect to the effect of $\mathrm{MgCl}_{2}$ concentration in the feed. Consistent with the 
result of effect of temperature, resistance contributed by membrane layer was also much higher than the one provided by stagnant layer as suggested from salt permeabilies in both layers. This suggests the accumulation of salt in the membrane surface due to salt transport was controlled by the membrane layer.

The work done by Dubas and Schlenoff [39] is very important to explain the behavior of structure-relation membrane transport. As seen in Table 2, when the $\mathrm{NaCl}$ concentration increased, the membrane thickness of PDAC/PSS layer decreased. From their work, this thickness decrease was evidenced and reached the maximum decrease at $\mathrm{NaCl}$ concentration of $0.1 \mathrm{M}$ and then started to increase again gradually when the concentration increased above $0.1 \mathrm{M}$. Another interesting result from their work was that there was no change in RMS roughness of the layer when the layer was exposed to $\mathrm{NaCl}$ concentration of $0.1 \mathrm{M}$, even though the layer was exposed for 400 min continuously.

Based on that investigation, the significant increase of membrane salt permeability (Ps) was contributed by the increase of salt concentration on the membrane surface as well as the decrease of the membrane thickness. These two results even more pronounce effect on the increase of membrane salt permeability compared to similar effect caused by temperature increase. Whereas, the decrease of stagnant layer salt permeability $\left(\mathrm{P}_{\mathrm{S}}\left({ }^{\delta}\right)\right)$ was owing to salt accumulation on the membrane surface causing more viscous flows and slower diffusion.

Table 2. The effect of $\mathrm{MgCl}_{2}$ Concentration on Membrane Salt Permeabilities, $\mathrm{P}_{\mathrm{S}}$ and Stagnant Layer Salt Permeabilities, $\mathrm{P}_{\mathrm{S}}{ }^{(\delta)}$ obtained as fitting parameters for Eq. 7

\begin{tabular}{lccc}
\hline$\left[\mathrm{MgCl}_{2}\right], \mathrm{ppm}$ & {$\left[\mathrm{MgCl}_{2}\right], \mathrm{M}$} & $\begin{array}{c}\mathrm{P}_{\mathrm{S}} \\
\mu \mathrm{m} / \mathrm{s}\end{array}$ & $\begin{array}{c}\mathrm{P}_{\mathrm{S}}^{(\delta)} \\
\mu \mathrm{m} / \mathrm{s}\end{array}$ \\
\hline $585.75 \pm 1.06$ & 0.0107 & 0.378 & 27.590 \\
$1026.75 \pm 2.47$ & 0.0188 & 0.668 & 25.565 \\
$4035.00 \pm 21.21$ & 0.0740 & 2.020 & 14.537 \\
\hline
\end{tabular}

Fig 5 illustrates the effect of salt concentration on membrane performance. As it was increased (as displayed by the direction of the arrow), the permeate flux also increased owing to the thickness decrease providing lower resistance to water molecules to pass through the membrane as previously explained. Meanwhile, the rejection rate significantly decreased owing to the increase of membrane salt permeability which promoted higher salt passage.

Comparing the effect of both parameters on membrane performance, it can be seen that temperature increases from approximately 20 to $40{ }^{\circ} \mathrm{C}$ resulting in the rise of permeate flux by $60 \%$ with only slight decrease in rejection rate. Meanwhile, when salt concentration increased from 500 to 4000 ppm, it caused the increase of permeate flux by $130 \%$ but coupled with significant decrease of salt rejection rate. This result suggested that the membrane stability deteriorated with the salt concentration. This is actually normal for typical commercial $\mathrm{NF}$ or RO membrane but it occurs at much higher concentration. The membrane structure deteriorates quickly when being exposed to higher concentration although in the case of our membrane, it is important to increase membrane stability by for instance, chemical crosslink.

\section{Conclusion}

In this work, the investigation of PEM TFC NF membrane on the effect of operating condition on its performance was done. Two important operating parameters namely, temperature and feed salt concentration were investigated; whereas two membrane performance indicators i.e. salt rejection rate and permeate flux were analyzed in response to the operating condition.

It can be concluded that the increase of both temperature and salt concentration could increase the water flux and decrease the salt rejection rate. However, temperature had less detrimental effect on membrane performance compared to salt concentration in the feed. This suggests that membrane stability must be improved, for example using chemical cross-linker and the membrane should be tested again at even higher salt concentration.

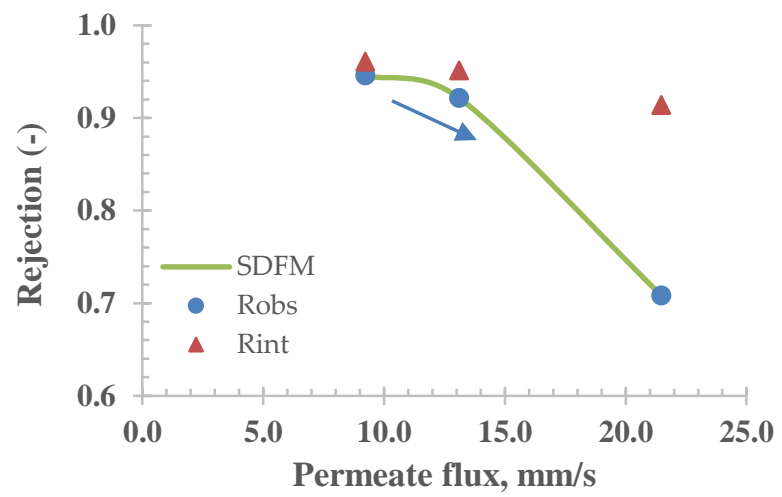

Fig 5. Fitting of Observable Rejection Rate experimental (circle) and intrinsic rejection rate (triangular) with SDFM (continuous line) (Testing condition: $\mathrm{P}$ $=10 \mathrm{bar}, \mathrm{MgCl}_{2}$ concentration $=500-4000 \mathrm{ppm}, \mathrm{CFV}=0.65 \mathrm{~m} / \mathrm{s}, \mathrm{T}=32.66$ $\pm 0.45^{\circ} \mathrm{C}$, and $\mathrm{pH}=6.42 \pm 0.17$ )

From membrane transport model point of view, it can be seen that the transport of salt and water across the membrane followed the modified solution diffusion model by including the coupling effect between ions within the stagnant layer, a thin film, adjacent to membrane surface in which concentration polarization occurred. Good agreement between SDFM and observed rejection rate $\left(\mathrm{R}_{\mathrm{obs}}\right)$ suggested that SDFM can be used to predict the performance of PEM NF membrane at least for single salt test and within the condition range used in this work. For future, it is then important to apply this model especially for the wider range of operating condition.

\section{Acknowledgements}

This research was funded by Deanship of Scientific Research of Al Imam Mohammad Ibn Saud Islamic University, Saudi Arabia, grant number 341403.

\section{References}

1. A. W. Mohammad, Y. H. Teow, W. L. Ang, Y. T. Chung, D. L. OatleyRadcliffe, N. Hilal, Nanofiltration membranes review: Recent advances and future prospects, Desalination. 356 (2015) 226-254

2. W. Cheng, C. Liu, T. Tong, R. Epsztein, M. Sun, R. Verduzco, et al., Selective removal of divalent cations by polyelectrolyte multilayer nanofiltration membrane: Role of polyelectrolyte charge, ion size, and ionic strength, J. Memb. Sci. 559 (2018) 98-106. 
3. A. Prasannan, J. Udomsin, H. C. Tsai, C. F. Wang, J. Y. Lai, Robust underwater superoleophobic membranes with bio-inspired carrageenan/laponite multilayers for the effective removal of emulsions, metal ions, and organic dyes from wastewater, Chem. Eng. J. (2019) 123585.

4. E. te Brinke, D. M. Reurink, I. Achterhuis, J. de Grooth, W. M. de Vos, Asymmetric polyelectrolyte multilayer membranes with ultrathin separation layers for highly efficient micropollutant removal, Appl. Mater. Today. (2019) 100471.

5. D. L. Shaffer, J. R. Werber, H. Jaramillo, S. Lin, M. Elimelech, Forward osmosis: Where are we now?, Desalination. 356 (2015) 271-284.

6. G. Decher, Fuzzy nanoassemblies: Toward layered polymeric multicomposites, Science. 277 (1997) 1232-1237.

7. J. L. Stair, J. J. Harris, M. L. Bruening, Enhancement of the iontransport selectivity of layered polyelectrolyte membranes through cross-linking and hybridization, Chem. Mater. 13 (2001) 2641-2648.

8. B. W. Stanton, J. J. Harris, M. D. Miller, M. L. Bruening, Ultrathin, Multilayered polyelectrolyte films as nanofiltration membranes, Langmuir. 19 (2003) 7038-7042.

9. R. Malaisamy, M. L. Bruening, High-flux nanofiltration membranes prepared by adsorption of multilayer polyelectrolyte membranes on polymeric supports, Langmuir. 21 (2005) 10587-10592.

10. S. U. Hong, R. Malaisamy, M. L. Bruening, Separation of fluoride from other monovalent anions using multilayer polyelectrolyte nanofiltration membranes, Langmuir. 23 (2007) 1716-1722.

11. S. U. Hong, L. Ouyang, M. L. Bruening, Recovery of phosphate using multilayer polyelectrolyte nanofiltration membranes, J. Memb. Sci. 327 (2009) 2-5.

12. J. J. Harris, J. L. Stair, M. L. Bruening, Layered polyelectrolyte films as selective, ultrathin barriers for anion transport, Chem. Mater. 12 (2000) 1941-1946.

13. L. Y. Ng, A. W. Mohammad, C. Y. Ng, C. P. Leo, R. Rohani, Development of nanofiltration membrane with high salt selectivity and performance stability using polyelectrolyte multilayers, Desalination. 351 (2014) 19-26.

14. Y. Huang, J. Sun, D. Wu, X. Feng, Layer-by-layer self-assembled chitosan/PAA nanofiltration membranes, Sep. Purif. Technol. 207 (2018) 142-150.

15. D. Wu, Y. Huang, S. Yu, D. Lawless, X. Feng, Thin film composite nanofiltration membranes assembled layer-by-layer via interfacial polymerization from polyethylenimine and trimesoyl chloride, J. Memb. Sci. 472 (2014) 141-153.

16. C. Liu, L. Shi, R. Wang, Crosslinked layer-by-layer polyelectrolyte nanofiltration hollow fiber membrane for low-pressure water softening with the presence of SO42- in feed water, J. Memb. Sci. 486 (2015) 169-176.

17. D. Menne, J. Kamp, J. Erik Wong, M. Wessling, Precise tuning of salt retention of backwashable polyelectrolyte multilayer hollow fiber nanofiltration membranes, J. Memb. Sci. 499 (2016) 396-405.

18. S. Rajesh, Y. Zhao, H. Fong, T. J. Menkhaus, Polyacrylonitrile nanofiber membranes modified with ionically crosslinked polyelectrolyte multilayers for the separation of ionic impurities, Nanoscale. 8 (2016) 18376-18389.

19. J. B. Schlenoff, S. T. Dubas, T. Farhat, Sprayed polyelectrolyte multilayers, Langmuir. 16 (2000) 9968-9969.

20. P.A. Chiarelli, M. S. Johal, J. L. Casson, J. B. Roberts, J. M. Robinson, H.L. Wang, Controlled fabrication of polyelectrolyte multilayer thin films using spin-assembly, Adv. Mater. 13 (2001) 1167-1171.

21. J. R. Kovacs, C. Liu, P. T. Hammond, Spray layer-by-layer assembled clay composite thin films as selective layers in reverse osmosis Membranes., ACS Appl. Mater. Interfaces. 7 (2015) 13375-13383

22. F. Fadhillah, A. M. Alghamdi, M. D. Alsubei, S. A. Aljlil, Synthesis of protein-fouling-resistance polyelectrolyte multilayered nanofiltration membranes through spin-assisted layer-by-layer assembly, J. King Saud Univ. - Eng. Sci. (2020).

23. L. Krasemann, B. Tieke, Selective Ion Transport across Self-Assembled Alternating Multilayers of Cationic and Anionic Polyelectrolytes, Langmuir. 16 (2000) 287-290.

24. F. Fadhillah, S. M. J. Zaidi, Z. Khan, M. M. Khaled, F. Rahman, P. T. Hammond, Development of polyelectrolyte multilayer thin film composite membrane for water desalination application, Desalination. 318 (2013) 19-24.

25. L. Pérez, I. Escudero, M. J. Arcos-Martínez, J. M. Benito, Application of the solution-diffusion-film model for the transfer of electrolytes and uncharged compounds in a nanofiltration membrane, J. Ind. Eng. Chem. 47 (2017) 368-374.

26. K. S. Spiegler, O. Kedem, Thermodynamics of hyperfiltration (reverse osmosis): criteria for efficient membranes, Desalination. 1 (1966) 311326.

27. J. G. Wijmans, The role of permeant molar volume in the solutiondiffusion model transport equations, J. Memb. Sci. 237 (2004) 39-50.

28. W. R. Bowen, H. Mukhtar, Characterisation and prediction of separation performance of nanofiltration membranes, J. Memb. Sci. 112 (1996) 263-274.

29. Y. Lanteri, P. Fievet, A. Szymczyk, Evaluation of the steric, electric, and dielectric exclusion model on the basis of salt rejection rate and membrane potential measurements, J. Colloid Interface Sci. 331 (2009) $148-155$.

30. A. Yaroshchuk, X. Martínez-Lladó, L. Llenas, M. Rovira, J. de Pablo, Solution-diffusion-film model for the description of pressure-driven trans-membrane transfer of electrolyte mixtures: One dominant salt and trace ions, J. Memb. Sci. 368 (2011) 192-201.

31. K. S. Kim, K. H. Lee, K. Cho, C. E. Park, Surface modification of polysulfone ultrafiltration membrane by oxygen plasma treatment, J. Memb. Sci. 199 (2002) 135-145.

32. F. Fadhillah, S. M. Javaid Zaidi, Z. Khan, M. M. Khaled, P. T. Hammond, Reverse osmosis desalination membrane formed from weak polyelectrolytes by spin assisted layer by layer technique, Desalin. Water Treat. 34 (2011) 44-49.

33. Q. Liu, F. Lin, R. A. Hartwick, Poly(diallyldimethylammonium chloride) as a Cationic Coating for Capillary Electrophoresis, J. Chromatogr. Sci. 35 (1997) 126-130.

34. J. E. Yoo, K. S. Lee, A. Garcia, J. Tarver, E. D. Gomez, K. Baldwin, et al., Directly patternable, highly conducting polymers for broad applications in organic electronics, Proc. Natl. Acad. Sci. 107 (2010) 5712 LP-5717.

35. C. Wu, L. Zhao, Y. Zhang, pH-Responsive nanofiltration membranes based on porphyrin supramolecular self-assembly by layer-by-layer technique, RSC Adv. 7 (2017) 47397-47406.

36. R. Salcedo-Díaz, P. García-Algado, M. García-Rodríguez, J. Fernández-Sempere, F. Ruiz-Beviá, Visualization and modeling of the polarization layer in crossflow reverse osmosis in a slit-type channel, J. Memb. Sci. 456 (2014) 21-30.

37. K. Köhler, H. Möhwald, G. B. Sukhorukov, Thermal behavior of polyelectrolyte multilayer microcapsules: 2. insight into molecular mechanisms for the PDADMAC/PSS system, J. Phys. Chem. B. 110 (2006) 24002-24010.

38. H. L. Tan, M. J. McMurdo, G. Pan, P. G. Van Patten, Temperature dependence of polyelectrolyte multilayer assembly, Langmuir. 19 (2003) 9311-9314.

39. S. T. Dubas, J. B. Schlenoff, Swelling and smoothing of polyelectrolyte multilayers by salt, Langmuir. 17 (2001) 7725-7727. 